\title{
EPAS1 gene variants are associated with sprint/ power athletic performance in two cohorts of European athletes
}

\author{
Sarah Voisin ${ }^{1}$, Pawel Cieszczyk ${ }^{2,4}$, Vladimir P Pushkarev ${ }^{3}$, Dmitry A Dyatlov ${ }^{3}$, Boris F Vashlyayev ${ }^{3}$, \\ Vladimir A Shumaylov ${ }^{3}$, Agnieszka Maciejewska-Karlowska ${ }^{4}$, Marek Sawczuk ${ }^{4}$, Lidia Skuza ${ }^{5}$, Zbigniew Jastrzebski ${ }^{2}$, \\ David J Bishop ${ }^{1}$ and Nir Eynon ${ }^{1,6^{*}}$
}

\begin{abstract}
Background: The endothelial PAS domain protein 1 (EPAS1) activates genes that are involved in erythropoiesis and angiogenesis, thus favoring a better delivery of oxygen to the tissues and is a plausible candidate to influence athletic performance. Using innovative statistical methods we compared genotype distributions and interactions of EPAS1 SNPs rs1867785, rs11689011, rs895436, rs4035887 and rs1867782 between sprint/power athletes $(n=338)$, endurance athletes $(n=254)$, and controls (603) in Polish and Russian samples. We also examined the association between these SNPs and the athletes' competition level ('elite' and 'sub-elite' level). Genotyping was performed by either Real-Time PCR or by Single-Base Extension (SBE) method.
\end{abstract}

Results: In the pooled cohort of Polish and Russian athletes, 1) rs1867785 was associated with sprint/power athletic status; the AA genotype in rs 1867785 was underrepresented in the sprint/power athletes, 2) rs11689011 was also associated with sprint/power athletic status; the $\Pi$ genotype in rs11689011 was underrepresented sprint/power athletes, and 3) the interaction between rs1867785, rs11689011, and rs4035887 was associated with sprint/power athletic performance; the combinations of the AA genotype in rs4035887 with either the AG or GG genotypes in rs1867785, or with the CT or CC genotypes in rs11689011, were underrepresented in two cohorts of sprint/power athletes.

Conclusions: Based on the unique statistical model rs1867785/rs11689011 are strong predictors of sprint/power athletic status, and the interaction between rs1867785, rs11689011, and rs4035887 might contribute to success in sprint/power athletic performance.

Keywords: Athletic performance, EPAS1, Genomics, Genes, Endurance, Sprint

\section{Background}

Maximal oxygen uptake $\left(\mathrm{VO}_{2 \max }\right)$ refers to the highest rate at which oxygen can be consumed by the body during intense exercise [1], and is, among other factors, an important predictor of elite endurance performance [2]. Studies have shown that the changes in $\mathrm{VO}_{2 \max }$ following exercise training vary markedly between individuals and that $\sim 50 \%$ of the variance can be explained by

\footnotetext{
* Correspondence: nir.eynon@vu.edu.au

'Institute of Sport, Exercise and Active Living (ISEAL), Victoria University,

Melbourne, Australia

${ }^{6}$ Murdoch Childrens Research Institute, The Royal Children's Hospital,

Melbourne, Australia

Full list of author information is available at the end of the article
}

genetic factors [3]. Over the last two decades, many Single Nucleotide Polymorphisms (SNPs) have been suggested to influence elite performance and the variability in $\mathrm{VO}_{2 \max }$ increase following exercise training [4]. While most of these SNPs were discovered using the candidate gene approach [5], a more comprehensive, genome-wide linkage approach has identified a genomic region on chromosome 2 that is associated with the $\mathrm{VO}_{2 \max }$ training response. The endothelial PAS domain protein 1 (EPAS1) gene was one of the four genes in this region responsible for this linkage [6].

EPAS1 is a transcription factor playing a key role in the Hypoxia Inducible Factor (HIF) pathway in blood, 
which is responsible for activating gene expression in response to hypoxia [7]. In normal oxygen conditions, EPAS1 is quickly degraded in the cytoplasm. However, when oxygen levels drop, EPAS1 becomes stabilized, translocates to the nucleus and activates genes that are involved in erythropoiesis (e.g. erythropoietin), and angiogenesis (e.g., vascular endothelial growth factor), thus favoring a better delivery of oxygen to the tissues [8]. Delivery of oxygen to skeletal muscles during endurance exercise is viewed a factor limiting $\mathrm{VO}_{2 \max }$ [1]. Therefore, as a hypoxia detector and as an activator of improved oxygen delivery to the active tissues, EPAS1 is a plausible candidate to influence endurance performance.

EPAS1 SNPs have been previously associated with blood parameters, such as alterations in erythropoietin, hemoglobin and hematocrit [9-11], that are important for success in athletic performance. For instance, Tibetans with the TT genotype in registered SNP (rs) 11689011 had lower hemoglobin concentration compared with their TC counterparts [9]. However, to date, only one study has examined a possible link between SNPs within EPAS1 and elite athletic performance [12]. This study looked at SNPs and haplotypes within EPAS1 in elite Australian athletes, stratified to two groups participating in middledistance (from $50 \mathrm{~s}$ to $10 \mathrm{~min}, \mathrm{n}=242$ ), and long-distance (from $\sim 2$ to $10 \mathrm{~h}, \mathrm{n}=151$ ) events. These groups were compared to a non-athletic control group. The $\mathrm{T}$ allele in rs11689011 and the G allele in rs1867785, two SNPs located in the first intron of EPAS1, were overrepresented in the group of endurance athletes compared with controls [12]. Furthermore, in the same study, two haplotypes involving rs1867785, rs11689011, rs895436 and rs4035887 were associated with elite endurance performance. While haplotype G (A-T-G-G) was overrepresented in elite endurance athletes, haplotype F (G-C-C-G) was underrepresented in elite endurance athletes compared to controls [12]. Despite these positive findings, and the strong biological rational behind investigating EPAS1 in relation to elite endurance performance, this is the only genetic association study showing that EPAS1 SNPs impact performance, and the sprinters consisting primarily of 100-400 m track runners and sprint cyclists were excluded from the analysis. Replication studies are therefore needed to confirm this association, particularly in different populations, and with a larger sample size [13].

Therefore, the aim of this study was to compare genotype distributions and interactions of the EPAS1 SNPs rs1867785, rs11689011, rs895436, rs4035887 and rs1867782 between sprint/power athletes, endurance athletes, and controls in Polish and Russian cohorts. We also examined the association between the EPAS1 SNPs and athletic status according to the athletes' level of competition ('elite' and 'sub-elite' level). In light of the relationship previously observed between endurance-related phenotypes and SNPs in the EPAS1, we hypothesized that EPAS1 SNPs would be associated with elite endurance performance compared to controls and sprint/power athletes. We did not have specific directional hypotheses for rs895436, rs4035887 and rs1867782 as these SNPs have not previously been associated with any performance and/or endurance-related phenotypes. We did not have any directional hypothesis for rs11689011 either, due to the conflicting results reported for this SNP (the $\mathrm{T}$ allele in rs11689011 was associated with endurance athletic status in elite Australians on one hand, and with lower hemoglobin concentrations in Tibetans, on the other hand) $[9,12]$. However, in line with the findings of Henderson et al. [12] we did expect the rs $1867785 \mathrm{G}$ allele to be associated with elite endurance performance. Finally, we did not have any directional hypotheses for any of the SNPs in relation to sprint/power performance.

\section{Methods}

The study was approved by the Pomeranian Medical University Ethics Committee, Poland, and the Ural State University of Physical Culture, Russia, and written informed consent was obtained from each participant. The study complied with the guidelines set out in the Declaration of Helsinki and the ethics policy of the Szczecin University [14].

\section{Participants}

The athletes and controls were all European Caucasians. The athletes were categorized as either endurance athletes or sprint/power athletes as determined by the distance, duration and energy requirements of their event/sport. All athletes were ranked in the top 10 nationally in their sport discipline and grouped as being either 'elite-level' or 'subelite' based on their best personal performance. Those in the elite group had participated in international competitions such as World and European Championships, and/ or Olympic Games, whereas those in the sub-elite group had participated in national competitions only. Details on the number of participants in the elite and sub-elite group are presented in Table 1 .

\section{Polish sample}

The sample comprised 198 Polish athletes (all men; mean age \pm SD, $28 \pm 4.4 \mathrm{y}$ ), including 92 elite and sub-elite sprint/power athletes and 106 elite and sub-elite endurance athletes, as well as 428 healthy, unrelated, sedentary controls (all male students of the University of Szczecin; mean age $\pm \mathrm{SD}, 20.8 \pm 1.2 \mathrm{y}$ ).

\section{Russian sample}

The Russian sample comprised 394 athletes (287 men and 107 women; mean age \pm SD, $27.8 \pm 9.7$ y), including 246 elite and sub-elite sprint/power athletes and 148 elite and 
Table 1 Athletes' description

\begin{tabular}{|c|c|c|c|c|}
\hline & \multicolumn{2}{|c|}{$\begin{array}{l}\text { Polish athletes } \\
(n=196)\end{array}$} & \multicolumn{2}{|c|}{$\begin{array}{l}\text { Russian athletes } \\
(\mathrm{n}=394)\end{array}$} \\
\hline & $\begin{array}{l}\text { Elite } \\
(n=122)\end{array}$ & $\begin{array}{l}\text { Sub-elite } \\
(n=74)\end{array}$ & $\begin{array}{l}\text { Elite } \\
(n=131)\end{array}$ & $\begin{array}{l}\text { Sub-elite } \\
(n=263)\end{array}$ \\
\hline \multicolumn{5}{|l|}{ ENDURANCE } \\
\hline Rowing & 33 & 8 & 7 & 7 \\
\hline Swimming $800 / 1500 \mathrm{~m}$ & 1 & 9 & 1 & 2 \\
\hline Cycling & 11 & 3 & 0 & 0 \\
\hline Skating 3000/5000/10000 m & 0 & 0 & 9 & 28 \\
\hline Cross-country skiing & 2 & 0 & 2 & 62 \\
\hline Canoeing & 9 & 1 & 0 & 0 \\
\hline Walking & 0 & 0 & 5 & 9 \\
\hline Triathlon & 2 & 3 & 0 & 0 \\
\hline Pentathlon & 0 & 0 & 0 & 3 \\
\hline Decathlon & 0 & 0 & 0 & 10 \\
\hline Marathon & 0 & 6 & 0 & 0 \\
\hline Running 1500/3000/5000 m & 7 & 11 & 1 & 2 \\
\hline Total & 64 & 40 & 25 & 123 \\
\hline \multicolumn{5}{|l|}{ SPRINT/POWER } \\
\hline Skating 500/1000 m & 1 & 0 & 6 & 17 \\
\hline Weightlifting & 22 & 20 & 44 & 43 \\
\hline Long jump & 5 & 3 & 1 & 0 \\
\hline Sprint 100/200/400 m & 25 & 9 & 1 & 4 \\
\hline Swimming 50/100 m & 2 & 0 & 5 & 12 \\
\hline Shooting & 1 & 0 & 0 & 0 \\
\hline Pole vault & 1 & 2 & 0 & 3 \\
\hline Javelin throw & 1 & 0 & 0 & 0 \\
\hline Ice hockey & 0 & 0 & 27 & 16 \\
\hline Taekwondo & 0 & 0 & 3 & 5 \\
\hline Karate & 0 & 0 & 5 & 3 \\
\hline Boxing & 0 & 0 & 9 & 27 \\
\hline Wrestling & 0 & 0 & 3 & 8 \\
\hline Ski cross freestyle & 0 & 0 & 2 & 0 \\
\hline Snowboarding & 0 & 0 & 0 & 1 \\
\hline Discus throw & 0 & 0 & 0 & 1 \\
\hline Total & 58 & 34 & 106 & 140 \\
\hline
\end{tabular}

sub-elite endurance athletes, as well as 175 healthy unrelated sedentary controls (104 men and 44 women, all students or employees of the Ural State University of Physical Culture; mean age \pm SD, $30.2 \pm 10.7$ y). The description of the Polish and the Russian athletes according to their event/sport is summarized in Table 1.

\section{Genotyping}

Polish sample

Genomic DNA was isolated from buccal epithelium using GenElute Mammalian Genomic DNA Miniprep
Kit (Sigma, Hamburg, Germany) according to the manufacturer's instructions. All genetic analyses were performed at the Molecular Biology and Biotechnology Center, Faculty of Biology, University of Szczecin. All samples were genotyped in duplicate using allelic discrimination assays with Taqman ${ }^{\circ}$ probes (Applied Biosystems, Carlsbad, California, USA) on a CFX96 Touch $^{\mathrm{mm}}$ Real-Time PCR Detection System (Bio-Rad, Hercules, California, USA). To discriminate EPAS1 rs1867782, rs1867785, rs11689011, rs895436 and rs4035887 alleles, TaqMan ${ }^{\circ}$ Pre-Designed SNP Genotyping Assays were used (assay IDs: C_11639978_1, C__11639984_10, C___2148918_10, C___2148915_10, C__2162989_10, respectively), including appropriate primers and fluorescently labeled (FAM and VIC) MGB $^{\text {ma }}$ probes to detect the alleles. Genotypes were assigned using all of the data from the study simultaneously.

\section{Russian sample}

Genomic DNA was isolated from buccal epithelium or peripheral blood, during the years 2011-2013, using the Diatom $^{\text {Tu }}$ DNA Prep kit (Cat. \# D 1025, IsoGene Lab Ltd, Russia). Genotyping of five selected SNPs was performed by Single-Base Extension (SBE) method. The sequence surrounding each SNP was obtained from the Genome Reference Consortium Human genome build 37 assembly from the Ensembl Project [15]. The Primer3web software v. 4.0.0 [16] was used for designing the PCR primers. PCR product range was $109-173 \mathrm{bp}$. SBE primers to detect rs895436, rs11689011, rs1867782, and rs1867785 were designed to anneal on the positive strand immediately adjacent to the single nucleotide variation sites. SBE primer for detection of rs4035887 was designed to anneal on the negative strand. To avoid any non-specific amplification and extension products, all primers were BLASTed against human genome reference sequence. Sets of preselected PCR primer pairs and SBE primers were screened for potential cross-reactivity by using AutoDimer software.

Multiplex PCR was performed in a volume of $15 \mu \mathrm{L}$ containing $1 \times$ PCR buffer, $1.0 \mathrm{mM} \mathrm{MgCl}_{2}, 0.2 \mathrm{mM}$ dNTPs, $0.7 \mu \mathrm{M}$ of each primer (5 pairs), 1 unit SmarTaq DNA polymerase (Dialat Ltd, Russia) and $5 \mathrm{ng}$ of template DNA. Thermal cycler conditions were: $95^{\circ} \mathrm{C}$ for $30 \mathrm{~s}, 30$ cycles of $95^{\circ} \mathrm{C}$ for $45 \mathrm{~s}, 60^{\circ} \mathrm{C}$ for $45 \mathrm{~s}, 72^{\circ} \mathrm{C}$ for $60 \mathrm{sec}$ and finally $10 \mathrm{~min}$ at $72^{\circ} \mathrm{C}$ in GeneAmp ${ }^{\circ}$ PCR System 9700 (Applied Biosystems). Multiplex PCR products were checked for quality and yield by running $3 \mu \mathrm{l}$ in $2 \%$ agarose-TBE gels. $5 \mu \mathrm{L}$ of PCR products were cleaned with 1 unit of FastAP Thermosensitive Alkaline Phosphatase (TAP) and 10 units of Exonuclease I (both enzymes from Fermentas). Multiplex SBE reaction was performed by using SNaPshot ${ }^{\circ}$ Multiplex Kit (Applied Biosystems) in $5 \mu \mathrm{L}$ final volume, including $2.5 \mu \mathrm{L}$ of SnaPshot Multiplex Ready Reaction Mix, $1.0 \mu \mathrm{l}$ pooled SBE primers and 
$1.5 \mu \mathrm{l}$ of cleaned PCR product (the PCR sequences, the SBE primers and their final concentration can be received from the authors by request). The cycling conditions were $96^{\circ} \mathrm{C} 10 \mathrm{~s}, 50^{\circ} \mathrm{C} 5 \mathrm{~s}$ and $60^{\circ} \mathrm{C} 30 \mathrm{~s}$, during 25 cycles in GeneAmp ${ }^{\circ}$ PCR System 9700 (Applied Biosystems). To remove the unincorporated ddNTPs, the final product was incubated with 1 unit of TAP (Fermentas). SnaPshot products with GeneScan ${ }^{\mathrm{TM}}$ - $120 \mathrm{LIZ}^{\mathrm{TM}}$ Size Standard (AB) were diluted in $\mathrm{Hi}-\mathrm{Di}^{\mathrm{im}}$ Formamide $(\mathrm{AB})$, denatured and separated using an ABI PRISM 310 Genetic Analyzer (AB) with a $47 \mathrm{~cm}$ length capillary and $\mathrm{POP}-4^{\mathrm{TM}}$ polymer $(\mathrm{AB})$. The SnaPshot ${ }^{\oplus}$ Primer Focus $^{\oplus}$ Kit $(\mathrm{AB})$ was used to analyze individual SBE primers for their approximate sizing locations prior to performing the multiplex SBE reaction. Final data were analyzed using the GeneMapper ${ }^{\circledR}$ Software v. 4.1 from Applied Biosystems following the software manual.

K562 DNA High Molecular Weight from Promega Corp. (USA) served as positive control sample. Genetic profile of K562 DNA was following: rs895436 - G/G, rs11689011 - C/C, rs4035887 - T/T (for the negative strand), rs1867785 - G/G, rs1867782 - C/C.

Each of the five PCR products (for the five different SNPs) was formed from five different individuals, and was sequenced in separate reaction using BigDye ${ }^{\oplus}$ Terminator v3.1 Cycle Sequencing Kit (Applied Biosystems) with full coincidence of expected and observed sequences. Sequencing was performed in a second laboratory (Gordiz Ltd. Laboratory, Moscow, Russia), according to latest recommendations [17].

\section{Genotyping reliability across two laboratories}

Genotyping was performed in duplicate in the same Laboratory for accuracy. Two independent investigators have called the genotyping score in each laboratory-100\% of the genotypes could be called. For the purpose of results reliability across two laboratories in two different countries (Russia and Poland), different DNA samples (one for each SNP, positive or negative controls) were shipped from Russia to Poland and were genotyped by TaqMan assays. The results of the genotyping were in $100 \%$ agreement across the two laboratories.

\section{Statistical analysis}

The genotype frequencies of all individual SNPs are presented in Additional file 1. Before looking at both the individual effects of the five EPAS1 SNPs and their interactions, we selected the best genetic model for each SNP. Then, SNP main effects, as well as SNP-SNP interactions, were investigated using Multivariate Adaptive Regression Splines (MARS), a nonparametric regression method [18] that has been successfully applied for detecting SNP-SNP interaction in several studies [19-22]. Finally, the odds ratios (OR) of being either a sprint/power athlete or an endurance athlete were calculated for each significant
SNP and significant interacting pairs of SNPs using the best genetic model for each SNP. Details on the steps that have been taken in the statistical analysis are shown in Figure 1.

\section{Hardy-Weinberg equilibrium (HWE)}

$X^{2}$ analysis was used to confirm if the control group, in each of the two samples, met HWE expectations. HWE was tested separately for each SNP.

\section{Selection of the best genetic model for each SNP}

Three inheritance models (dominant, recessive and additive model) were assessed in the pooled cohorts of sprint/power and endurance athletes (Polish and Russian) using the Wald test in logistic regression models, and the best model was selected based on the minimum p-value for each SNP. The athletic status was divided into two categories and encoded as a dummy variable: athlete (encoded as 1) and control (encoded as 0). To adjust for multiple comparisons, the false discovery rate was controlled using the Benjamini and Hochberg correction [23]. For consistency, the same genetic model was applied separately in the Russian and Polish groups. All of the following steps were performed once when comparing sprint/power athletes with controls, and once again when comparing endurance athletes with controls.

\section{Analysis of SNP main effects and SNP-SNP interactions}

Variable importance ranking for SNPs with strong Linkage Disequilibrium (LD) has been shown to be biased in previous studies [24,25]. Therefore, LD in the 5 SNPs was examined using $\mathrm{r}^{2}$ [26], and the pairs of SNPs displaying a strong LD $\left(\mathrm{r}^{2}>0.8\right)$ were identified (Additional file 2). Only rs11689011 and rs1867785 were in strong LD $\left(r^{2}=0.96\right)$. Consequently, two MARS models, all corrected for sex, were developed: one including rs11689011, rs895436, rs4035887 and rs1867782, and another independent MARS model including rs1867785, rs895436, rs4035887 and rs1867782. For simplicity, we have only reported the MARS model excluding rs11689011 in the Results section.

In MARS, the maximum number of basis functions was set at 100, and the maximum degree of interaction was set at 2. As MARS does not provide variable significance using p-values, each covariate selected by MARS was used as input into a logistic regression model to determine its significance. All non-significant covariates ( $p$-value $>0.05$ ) were excluded. To confirm the significance of the covariates identified by the logistic regression, and to rank their importance, we used the Bootstrap Inclusion Fraction (BIF) criterion [27]. We obtained 10000 MARS models using 10000 random bootstrap samples with replacement from the original data set. Then, we calculated the proportion of times that a significant variable appeared in the 10000 MARS models and called this number the BIF. A variable which is 


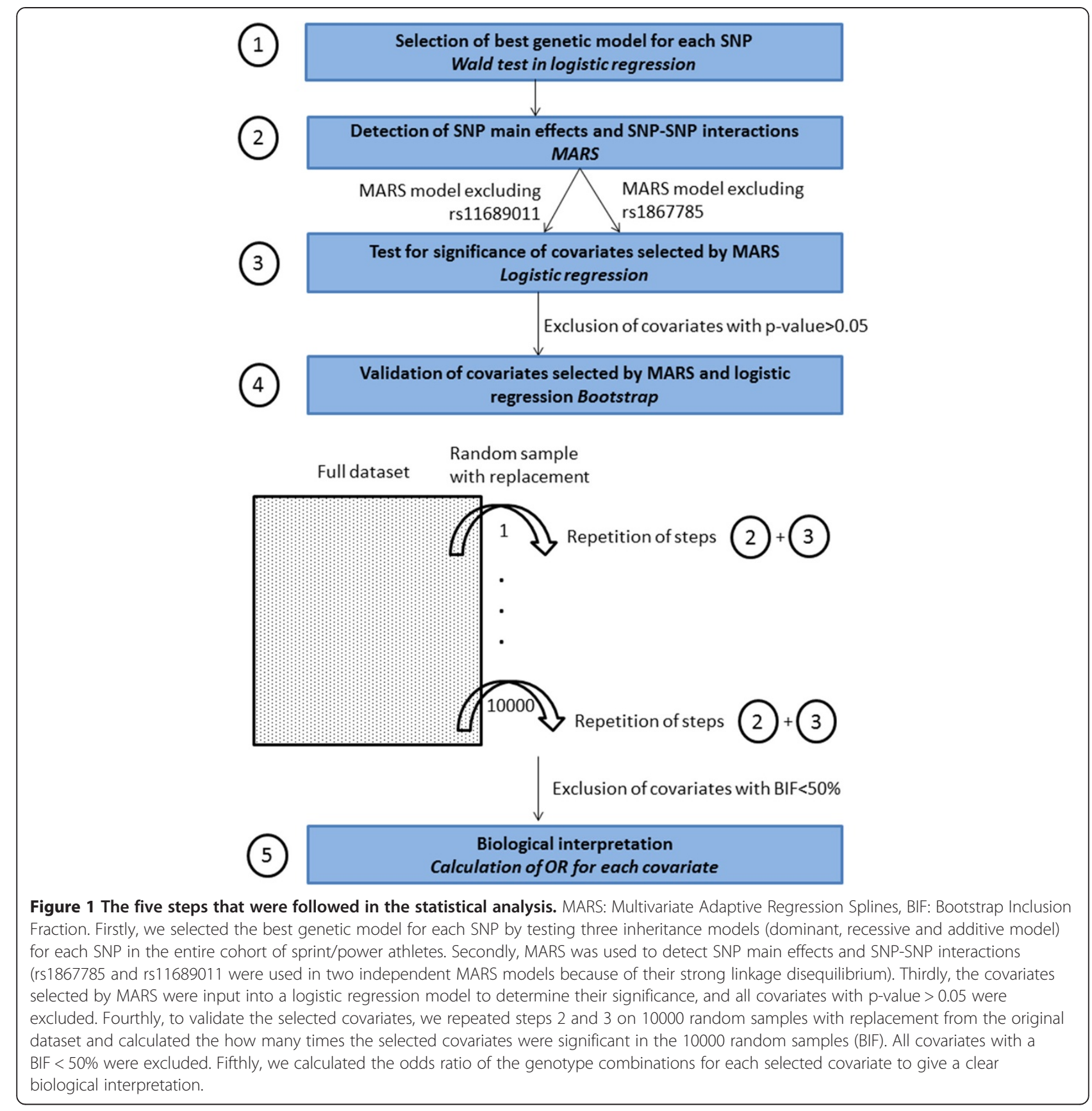

approximately uncorrelated with others, and is only significant at the chosen nominal $\alpha$ level in the MARS model, is selected in about $50 \%$ of bootstrap samples. As the p-value diminishes, the BIF tends toward $100 \%$. Thus, we only included covariates with a BIF $>50 \%$.

For each significant covariate, the odds ratio (OR) of being either a sprint/power or an endurance athlete, depending on the genotype, was calculated. The 95\% confidence intervals $(\mathrm{CI})$ were calculated by unconditional maximum likelihood estimation.

\section{Results}

In the pooled cohort of Russian and Polish controls, genotype distributions for each of the five SNPs was in agreement with HWE (p-value >0.05).

\section{Endurance athletes vs. controls}

In the Russian sample, the MARS model excluding rs11689011 yielded only one significant covariate, with a BIF $>50 \%$. An interaction between sex and rs 1867785 was observed: the GA and GG genotypes in rs1867785 
were underrepresented in women endurance athletes $(\mathrm{OR}=0.39$, Table 2$)$.

In the Polish sample, no covariate was selected in the MARS model excluding rs11689011. When combining the Russian and the Polish groups, no covariate was selected in the MARS model excluding rs11689011.

Using the MARS model excluding rs1867785, the results were almost identical to those of the MARS model excluding rs11689011 (Additional file 3: Table S2). However, in the Polish sample one significant covariate was retained with a BIF $>50 \%$. The TT genotype in rs11689011 was underrepresented in the cohort of endurance athletes ( $\mathrm{OR}=0.49$, Additional file 3: Table S2), especially in the elite-level cohort compared to their sub-elite counterparts $(\mathrm{OR}=0.31,95 \%$ confidence interval: 0.11-0.87, Table 3).

\section{Sprint/power athletes vs. controls}

In the Russian sample, the MARS model excluding rs11689011 yielded two significant covariates ( $\mathrm{p}$-value $<0.05$ in logistic regression); only one covariate, however, had a BIF $>50 \%$ (Table 2). The AA genotype in rs 1867785 was underrepresented in sprint/power athletes $(\mathrm{OR}=0.47$, Figure 2), especially in the elite sprint/power athletes compared to their sub-elite counterparts ( $\mathrm{OR}=0.35,95 \%$ confidence interval: 0.16-1.79, Table 3). In the Polish sample, no covariates were selected in the MARS model (Table 2).

In the Russian and Polish samples combined, the MARS model excluding rs11689011 yielded three significant covariates, two had a BIF $>50 \%$ (Table 2). The trend already observed for rs1867785 in the Russian sprint/power group was even stronger when combined with the Polish group
$(\mathrm{BIF}=90.1 \%)$; the AA genotype in rs1867785 was underrepresented in sprint/power athletes $(\mathrm{OR}=0.53$, Figure 2$)$, especially in elite sprint/power athletes compared to their sub-elite counterparts $(\mathrm{OR}=0.41,95 \%$ confidence interval: 0.22-0.75). Furthermore, an interaction between rs1867785 and rs4035887 was found; the combination of the AA genotype in rs4035887 and the GA or GG genotype in rs1867785 was underrepresented in sprint/power athletes ( $\mathrm{OR}=0.61$, Table 2 and Figure 3$)$. We note that the genotype distribution of individual SNPs does not provide information regarding the genotype distribution of their combinations. For example, $\mathrm{SNP}_{\mathrm{A}}$ and $\mathrm{SNP}_{\mathrm{B}}$ are two SNPs with alleles $\mathrm{A} / \mathrm{a}$ and $\mathrm{B} / \mathrm{b}$, respectively. In this example allele $\mathrm{A}$ is advantageous to performance, and allele B is also advantageous to performance but only when combined with allele a. From this pattern, one would expect allele A to be overrepresented in athletes, but the $\mathrm{A}+\mathrm{B}$ combination to be underrepresented in athletes.

Using the MARS model excluding rs1867785, the results were almost identical to those of the MARS model excluding rs11689011 (Additional file 3: Table S2). However, the interaction between rs11689011 and rs4035887 in the Russian and Polish sample combined was not significant $(\mathrm{BIF}=49.5 \%)$.

\section{Discussion}

We have examined the genotype distribution and SNP-SNP interaction of five SNPs in the first intron of the EPAS1 gene in European sprint/power and endurance athletes. We initially hypothesised that these SNPs would be associated with endurance athletic status. However, contrary to our

Table 2 Covariates identified in the MARS model excluding rs11689011

\begin{tabular}{|c|c|c|c|c|c|c|}
\hline & & Covariate & P-value ${ }^{1}$ & $\mathrm{BIF}^{2}$ & Odds Ratio & \\
\hline \multirow[t]{4}{*}{ Endurance athletes vs. controls } & Russians & rs $1867785^{*}$ sex & 0.00022 & 61.7 & Other combinations & 1 (ref) \\
\hline & & & & & GA or GG in women & $0.39(0.24-0.65)$ \\
\hline & Polish & & & & & \\
\hline & Russians + Polish & & & & & \\
\hline \multirow[t]{10}{*}{ Sprint/power athletes vs. controls } & Russians & rs4035887 & 0.0072 & 43.6 & GA or GG & 1 (ref) \\
\hline & & & & & AA & $0.54(0.34-0.88)$ \\
\hline & & rs1867785 & 0.0017 & 78.3 & GA or $\mathrm{GG}$ & 1 (ref) \\
\hline & & & & & AA & $0.47(0.25-0.84)$ \\
\hline & Polish & & & & & \\
\hline & Russians + Polish & rs1867785 & 0.00016 & 90.1 & $\mathrm{GA}+\mathrm{GG}$ & 1 (ref) \\
\hline & & & & & $\mathrm{AA}$ & $0.53(0.35-0.80)$ \\
\hline & & rs4035887*rs 1867785 & 0.00016 & 52.6 & Other combinations & 1 (ref) \\
\hline & & & & & AA at rs4035887 and & $0.61(0.45-0.85)$ \\
\hline & & & & & GA or GG at rs 1867785 & \\
\hline
\end{tabular}

${ }^{1}$ P-value obtained by logistic regression.

${ }^{2}$ Bootstrap Inclusion Fraction calculated after running 10000 MARS models on 10000 bootstrap samples. A BIF of 90.1 indicates that the covariate of interest was selected in $90.1 \%$ of the MARS models.

*denotes an interaction. 
Table 3 Genotype frequencies of the three Single Nucleotide Polymorphisms (SNPs) significantly associated with athletic performance

\begin{tabular}{|c|c|c|c|c|c|c|c|c|c|c|c|c|c|}
\hline \multirow[t]{2}{*}{ SNP } & \multirow{2}{*}{$\begin{array}{l}\text { Major/minor } \\
\text { allele }\end{array}$} & \multirow[t]{2}{*}{ Model } & \multirow[t]{2}{*}{ Genotypes } & \multicolumn{5}{|c|}{ Russians (Males + Females) } & \multicolumn{5}{|c|}{ Polish (Males) } \\
\hline & & & & $\begin{array}{l}\text { Controls } \\
(n=175)\end{array}$ & \multicolumn{2}{|c|}{$\begin{array}{l}\text { Endurance } \\
\text { athletes }(n=148)\end{array}$} & \multicolumn{2}{|c|}{$\begin{array}{l}\text { Sprint/power } \\
\text { athletes }(n=246)\end{array}$} & $\begin{array}{l}\text { Controls } \\
(n=428)\end{array}$ & \multicolumn{2}{|c|}{$\begin{array}{l}\text { Endurance } \\
\text { athletes }(n=106)\end{array}$} & \multicolumn{2}{|c|}{$\begin{array}{l}\text { Sprint/power } \\
\text { athletes }(n=92)\end{array}$} \\
\hline \multirow[t]{6}{*}{ rs11689011 } & $\mathrm{T} / \mathrm{C}$ & Recessive & TC or CC & $139(79.4 \%)$ & $119(80.4 \%)$ & & $220(89.4 \%$ & & $353(82.5 \%)$ & $96(90.6 \%)$ & & $82(89.1 \%)$ & \\
\hline & & & & & Elite & $18(72 \%)$ & Elite & 97 (91.5\%) & & Elite & $61(93.8 \%)$ & Elite & $53(91.4 \%)$ \\
\hline & & & & & Sub-elite & $101(82.1 \%)$ & Sub-elite & $123(87.9 \%)$ & & Sub-elite & 35 (85.4\%) & Sub-elite & $29(85.3 \%)$ \\
\hline & & & $\pi$ & $36(20.6 \%)$ & $29(19.6 \%)$ & & $26(10.6 \%)$ & & $75(17.5 \%)$ & $10(9.4 \%)$ & & $10(10.9 \%)$ & \\
\hline & & & & & Elite & $7(28 \%)$ & Elite & $9(8.5 \%)$ & & Elite & $4(6.2 \%)$ & Elite & $5(8.6 \%)$ \\
\hline & & & & & Sub-elite & $22(17.9 \%)$ & Sub-elite & $17(12.1 \%)$ & & Sub-elite & $6(14.6 \%)$ & Sub-elite & $5(14.7 \%)$ \\
\hline \multirow[t]{6}{*}{ rs4035887 } & $\mathrm{G} / \mathrm{A}$ & Dominant & GA or GG & $130(74.3 \%)$ & $119(80.4 \%)$ & & $207(84.1 \%$ & & $297(69.4 \%)$ & $68(64.2 \%)$ & & $62(67.4 \%)$ & \\
\hline & & & & & Elite & $19(76.0 \%)$ & Elite & $88(83.0 \%)$ & & Elite & $41(63.1 \%)$ & Elite & $37(63.8 \%)$ \\
\hline & & & & & Sub-elite & $100(81.3 \%)$ & Sub-elite & $119(85.0 \%)$ & & Sub-elite & $27(65.9 \%)$ & Sub-elite & $25(73.5 \%)$ \\
\hline & & & AA & 45 (25.7\%) & $29(19.6 \%)$ & & $39(15.9 \%)$ & & 131 (30.6\%) & $38(34.9 \%)$ & & $30(32.6 \%)$ & \\
\hline & & & & & Elite & $6(24.0 \%)$ & Elite & $18(17.0 \%)$ & & Elite & 24 (36.9\%) & Elite & $21(36.2 \%)$ \\
\hline & & & & & Sub-elite & $23(18.7 \%)$ & Sub-elite & $21(15.0 \%)$ & & Sub-elite & 14 (31.4\%) & Sub-elite & $9(26.5 \%)$ \\
\hline \multirow[t]{6}{*}{ rs1867785 } & $\mathrm{A} / \mathrm{G}$ & Recessive & GA or GG & $142(81.1 \%)$ & $122(82.4 \%)$ & & $222(90.2 \%$ & & $356(83.2 \%)$ & $96(90.6 \%)$ & & $82(89.1 \%)$ & \\
\hline & & & & & Elite & $18(72 \%)$ & Elite & $98(92.5 \%)$ & & Elite & 61 (93.8\%) & Elite & 53 (91.4\%) \\
\hline & & & & & Sub-elite & $104(84.6 \%)$ & Sub-elite & $124(88.6 \%)$ & & Sub-elite & 35 (85.4\%) & Sub-elite & $29(85.3 \%)$ \\
\hline & & & $\mathrm{AA}$ & 33 (18.9\%) & $26(17.6 \%)$ & & $24(9.8 \%)$ & & 72 (16.8\%) & $10(9.4 \%)$ & & $10(10.9 \%)$ & \\
\hline & & & & & Elite & $7(28 \%)$ & Elite & $8(7.5 \%)$ & & Elite & $4(6.2 \%)$ & Elite & 5 (8.6\%) \\
\hline & & & & & Sub-elite & 19 (15.4\%) & Sub-elite & 16 (11.4\%) & & Sub-elite & $6(14.6 \%)$ & Sub-elite & $5(14.7 \%)$ \\
\hline
\end{tabular}




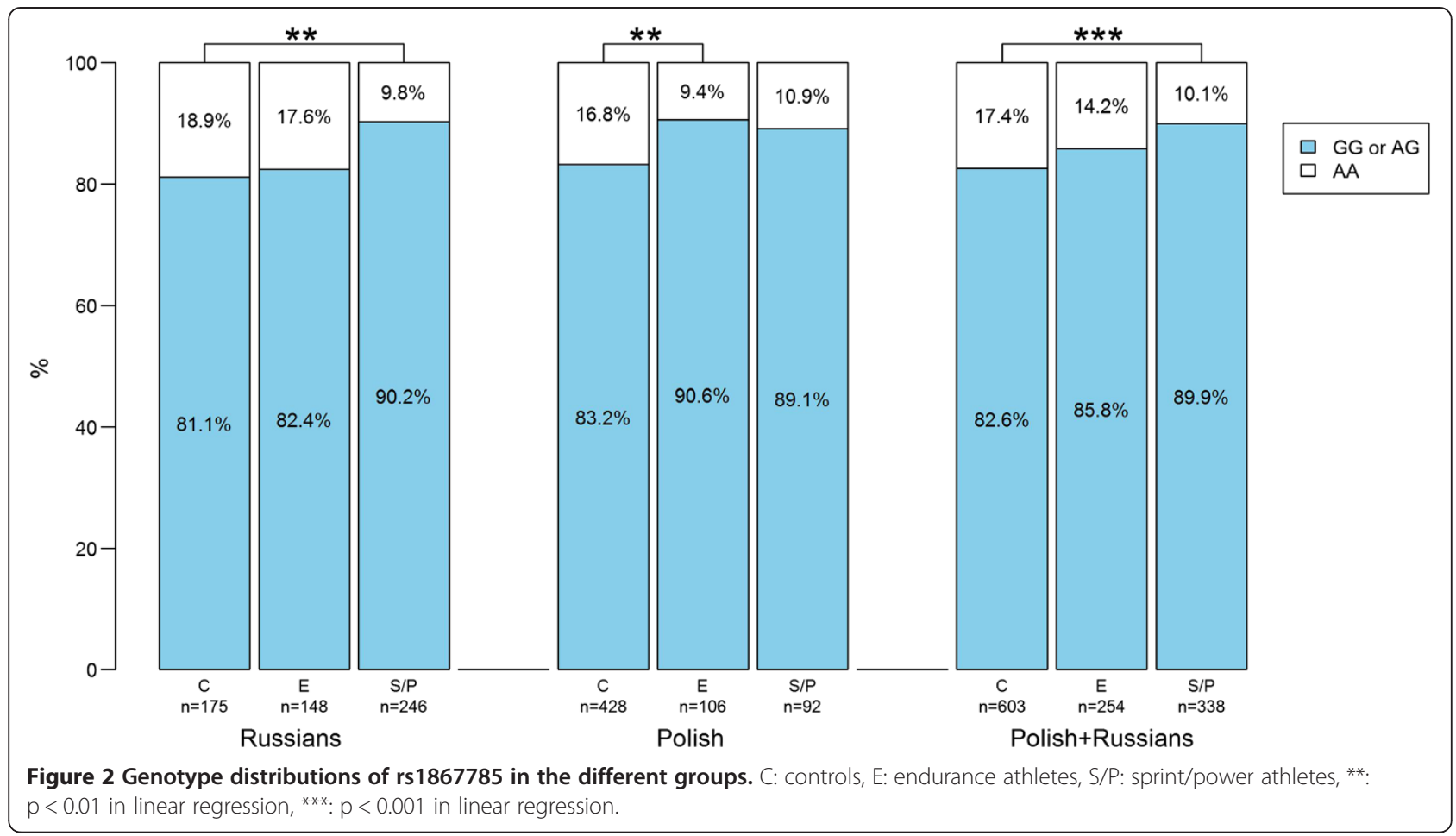

hypotheses our main findings were as follow: In the pooled cohort of Polish and Russian athletes, 1) rs1867785 was associated with sprint/power athletic status; the AA genotype in rs1867785 was underrepresented in sprint/power athletes, 2) rs11689011 was also associated with sprint/ power athletic status; the TT genotype in rs11689011 was underrepresented in sprint/power athletes, and 3) the interaction between rs1867785/rs11689011, and rs4035887 was associated with sprint/power athletic performance; the combinations of the AA genotype in rs4035887 with

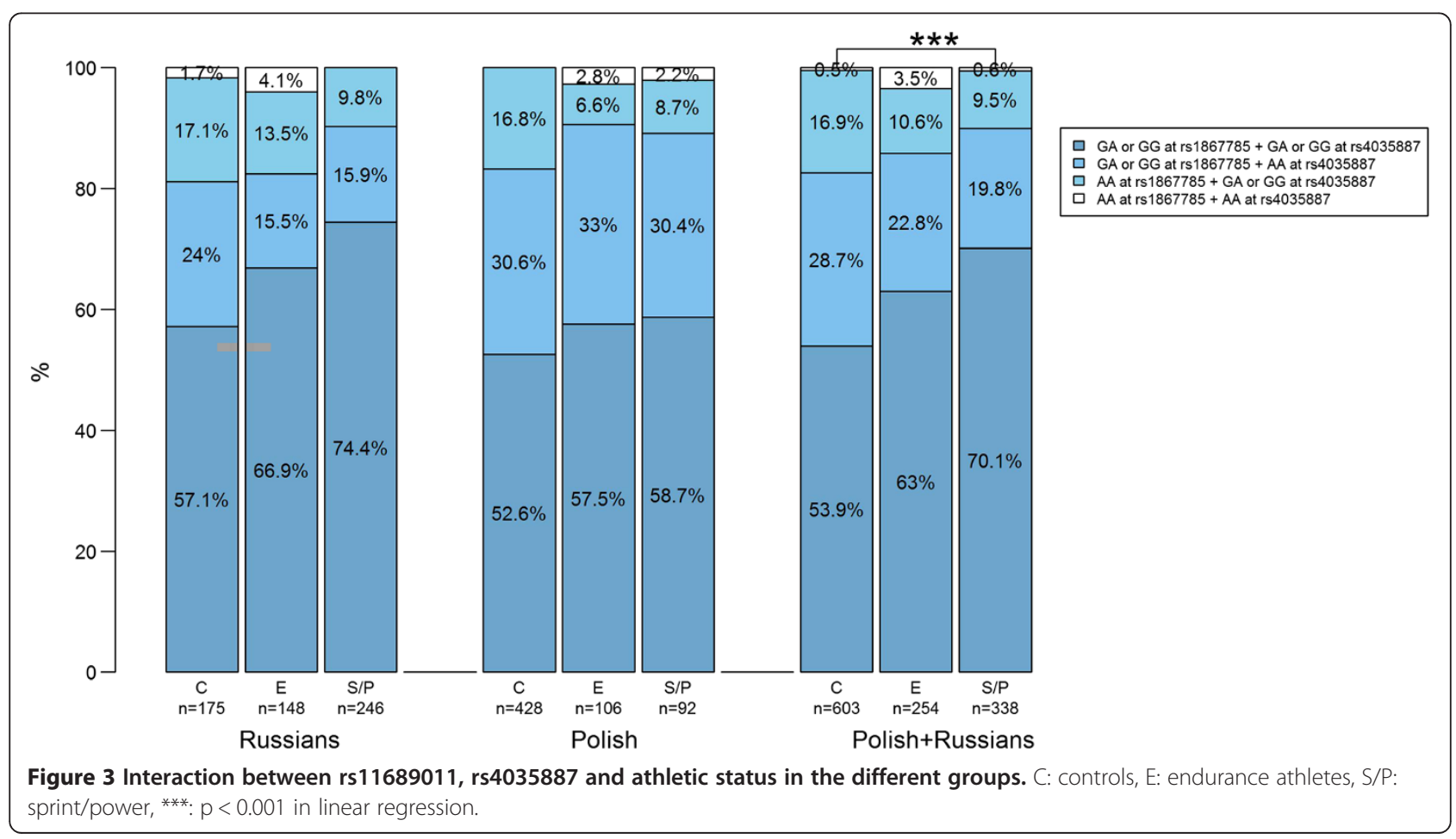


either the GA or GG genotypes in rs1867785, or with the CT or CC genotypes in rs11689011, were underrepresented in two cohorts of sprint/power athletes.

Compared with a previous study, the results of the present study were unexpected. Henderson et al. [12] have reported that the $\mathrm{G}$ allele in rs1867785 and the $\mathrm{T}$ allele in rs11689011 were overrepresented in endurance athletes, whereas we have observed that the $G$ allele in rs1867785 was underrepresented (and the A allele was overrepresented) in female Russian endurance athletes. Furthermore, in our cohort, the T allele in rs11689011 was overrepresented in female Russian endurance athletes, but underrepresented in male Polish endurance athletes. A possible explanation for the discrepancies between our study and the study by Henderson et al. [12] is that our results regarding rs11689011 and rs1867785 were sex-specific. Sex-specific effects of SNPs are common in a wide range of phenotypes such as waist-hip ratio [28], susceptibility to sport-related injury [29], and increased muscle strength in response to training [30]. We have also recently shown that PPARGC1A rs4697425 was associated with elite female, but not with male endurance running performance [31]. However, the Polish endurance sample only comprised males, while the Russian sample included both males and females. Interestingly, the cohort in the study by Henderson et al. included both males and females athletes, but sex was not investigated as a covariate in their analysis [12].

Looking at each SNP separately, we found that rs1867785 and rs11689011 were associated with sprint/power performance. However, these SNPs had very similar genotype distribution and are in strong $\operatorname{LD}\left(r^{2}=0.964\right)$; the A allele in rs1867785 segregates with the $\mathrm{T}$ allele in rs11689011. Since previous studies have suggested that SNPs with strong LD cannot be considered in the same statistical model [24,25], and only one of these SNPs might influence sprint/power performance, we have created two MARS models and considered only one of the SNPs in each model. As expected, the two MARS models yielded almost identical results in all cohorts. However, only rs11689011 was associated with endurance athletic status in the Polish sample, while this was not the case for rs1867785. This might be explained by the small difference in genotype distribution in rs11689011 and rs1867785 in the Polish control sample. In addition, the BIF obtained for rs11689011, when comparing Polish endurance athletes with controls, was not strong enough (65.4\%), indicating a correlation close to non-significance and sensitive to small changes in genotype frequencies. The relatively large sample size generated from studying two cohorts of athletes in the present study further reinforces the confidence in the results of the present study.

Indeed, in the present study two European Caucasian cohorts of athletes were grouped to explore the association between SNPs in EPAS1 and athletic performance.
Our previous results indicated that combining two cohorts of Caucasian athletes, especially when they are closelyrelated, would be useful approach to detect an association between SNPs and athletic status [13]. While previous studies combined athletes from different ancestries [32,33], here we have studied athletes from closely-related European ancestries (e.g., Polish and Russians). This is reinforced by the similarity in the genotype distribution in the control groups (no more than 5\% differences between the Russian and Polish controls). Furthermore, studying two cohorts of athletes has increased the sample size (overall 338 sprint/ power athletes and 254 endurance athletes), which further strengthened our results and the likelihood that these specific EPAS1 SNPs show a genuine association with elite sprint/power performance.

An additional novel finding in the present study is that the AA genotype in rs1867785, and the TT genotype in rs11689011, is even more underrepresented in elite sprint/ power athletes compared with their sub-elite counterparts. This has previously been demonstrated for the highlystudied ACTN3 R577X SNP, as the 577XX genotype was found in a lower frequency in elite sprint/power athletes compared to their national-level counterparts [34-36]. This observation indicates that while the EPAS1 SNPs are associated with the development of sprint/power ability, they might be even more important in the development of world-class sprint/power ability. This finding, along with all the other findings in the present study, was obtained using the Bootstrap Inclusion Fractions (BIF) statistical method, which as far as we are aware of, has never been used in sports genomics.

The BIF analysis is a useful technique for investigating variations among selected models in samples drawn at random with replacement. Such samples mimic datasets that are structurally similar to that under study and that could plausibly have arisen instead [37]. Initially designed to test the stability of multivariable models, this nonparametric method allowed us to test whether the EPAS1 SNPs selected by MARS were sensitive to small changes in the data, and confirm that they were unlikely to be false positives. Also, this method allowed us to see the relative importance of the different variables; while rs1867785 and rs11689011 showed very strong main effects in sprint/power athletes, their interaction with rs4035887 was of smaller importance.

We have also shown that several SNPs within EPAS1 are associated with endurance athletic status, in a sex-specific manner. The TT genotype in rs11689011 was underrepresented in the cohort of Polish endurance athletes. However, this association was demonstrated only when either the Polish or the Russian groups were analysed separately, and was abolished when the two cohorts were combined. We argue that we cannot be certain that these positive findings are not false positives, as they might be a limitation of the 
smaller sample size. On the other hand, our positive findings regarding the association between the EPAS1 SNPs and sprint/power athletic status were found in the combined cohort of sprint/power athletes, and the BIFs that were calculated for rs11689011 and rs1867785 were extremely high $(90.1 \%$ for rs 1867785 , and $93.6 \%$ for rs11689011). Furthermore, these associations were more pronounced when the athletes' level of competition was considered.

This study is not without limitations. In case-control studies, the relative proportions of controls and cases impacts the sample size required to detect an association with a given power and significance level. In the present study, with similar genotype distributions in Russians and Polish, to detect an association with the same effect size, at the same power and significance level, we would require a larger sample size in the Russian population. We acknowledge that the difference in numbers of athletes and controls in our study might therefore contribute to our results and the lack of replication in both athlete groups. However, in any association study with elite athletes it is a challenge to increase the sample size due to the very low number of elite athletes available to study.

Finally, in previous reports EPAS1 SNPs have demonstrated an association with performance-related blood parameters (e.g., alterations in erythropoietin, hemoglobin and hematocrit) [9-11], and elite endurance performance [12], in humans. However, is has also been shown that EPAS1 deficient mice have greater oxidative stress and an impaired response to oxidative stress [38]. A reduction in hematocrit levels and a global decrease in peripheral blood counts have also been observed in EPAS1-null mice [39]. Although no specific SNPs were tested in the mice model, these studies illustrate the potential importance of the EPAS1 gene in athletic-related phenotypes.

\section{Conclusion}

We found an association between EPAS1 rs1867785 and EPAS1 rs11689011 and sprint/power athletic status, and an interaction between rs1867785/11689011 and rs4035887 and sprint/power athletic status in two cohorts of closelyrelated European athletes. Based on the statistical model used either rs1867785 or rs11689011 are related to sprint/ power athletic status. The association between rs1867785 and sprint/power athletic status is in line with a previous study in Australian athletes [12]. Unlike the vast number of investigations into the genetics of endurance performance, the genetic influence on elite sprint/power performance has received limited attention, and only a few studies have characterized the associations between genetic variants and elite sprint/power performance. Most studies to date have recruited only one cohort of athletes and were therefore hampered by insufficient sample size. In the present study, we have combined two cohorts of athletes and used innovative statistical methods, which provide confidence in our results. Functional studies directly demonstrating cause and effect, or providing any proposed cellular or molecular mechanisms to explain the association, are needed to extend and validate these findings.

\section{Additional files}

\section{Additional file 1: Genotype frequencies of the five investigated Single Nucleotide Polymorphisms (SNPs). \\ Additional file 2: Linkage disequilibrium (LD) map of the five investigated SNPs in EPAS1. The upper horizontal line represents the strand of chromosome 2 containing EPAS1 and all five investigated SNPS. The triangle below indicates the pairwise LD $\left(r^{2}\right)$ between the five SNPS. Each SNP corresponds to a diagonal of this triangle, and the intersection of two diagonals contains the value of LD for the corresponding SNP pair. The colour within the squares represents the strength of the linkage between each pair. Of all possible pairs of SNPs, only rs1867785 and rs11689011 are in strong LD (red colour) \\ Additional file 3: Table S2. Covariates identified in the different MARS models.}

\section{Competing interests}

The authors declare that they have no competing interests.

\section{Authors' contributions}

SV and NE have made substantial contributions to conception and design, analysis and interpretation of data, and have been involved in drafting the manuscript or revising it critically for important intellectual content. PC and VPP conceived the study, participated in its design and coordination and helped drafting the manuscript. DAA and LS carried out the genetic studies and participated in its design and its data collection. BFV, VAS, AMK and MS carried out the genetic studies and participated in its design. ZJ carried out the molecular genetic studies. DJB has been involved in drafting the manuscript and revising it critically for important intellectual content. Al authors read and approved the final manuscript.

\section{Acknowledgements}

This study was supported by the Ministry of Sport of the Russian Federation, and Gordiz Ltd (Moscow, Russia).

\section{Author details}

'Institute of Sport, Exercise and Active Living (ISEAL), Victoria University, Melbourne, Australia. 'Department of Tourism and Recreation, Academy of Physical Education and Sport, Gdansk, Poland. ${ }^{3}$ Ural State University of Physical Culture, Chelyabinsk, Russia. ${ }^{4}$ Faculty of Physical Culture and Health Promotion, University of Szczecin, Szczecin, Poland. ${ }^{5}$ Cell Biology Department, Faculty of Biology, University of Szczecin, Szczecin, Poland. ${ }^{6}$ Murdoch Childrens Research Institute, The Royal Children's Hospital, Melbourne, Australia.

Received: 19 December 2013 Accepted: 7 May 2014 Published: 18 May 2014

\section{References}

1. Bassett DR, Howley ET: Limiting factors for maximum oxygen uptake and determinants of endurance performance. Med Sci Sports Exerc 2000, 32:70-84.

2. Bentley DJ, Newell J, Bishop D: Incremental exercise test design and analysis: implications for performance diagnostics in endurance athletes. Sports Med 2007, 37:575-586

3. Bouchard C, An P, Rice T, Skinner JS, Wilmore JH, Gagnon J, Pérusse L, Leon AS, Rao DC: Familial aggregation of VO2max response to exercise training: results from the HERITAGE Family Study. J Appl Physiol 1999, 87:1003-1008

4. Eynon N, Ruiz JR, Oliveira J, Duarte JA, Birk R, Lucia A: Genes and elite athletes: a roadmap for future research. J Physio/ 2011, 589:3063-3070. 
5. Wang G, Padmanabhan S, Wolfarth B, Fuku N, Lucia A, Ahmetov II, Cieszczyk P, Collins M, Eynon N, Klissouras V, Williams A, Pitsiladis Y: Genomics of elite sporting performance: what little we know and necessary advances. Adv Genet 2013, 84:123-149.

6. Bouchard C, Rankinen T, Chagnon YC, Rice T, Pérusse L, Gagnon J, Borecki I, An P, Leon AS, Skinner JS, Wilmore JH, Province M, Rao DC: Genomic scan for maximal oxygen uptake and its response to training in the HERITAGE family study. J Appl Physiol 2000, 88:551-559.

7. Patel SA, Simon MC: Biology of hypoxia-inducible factor-2alpha in development and disease. Cell Death Differ 2008, 15:628-634.

8. Ke Q, Costa M: Hypoxia-inducible factor-1 (HIF-1). Mol Pharmacol 2006, 70:1469-1480.

9. Beall CM, Cavalleri GL, Deng L, Elston RC, Gao Y, Knight J, Li C, Li JC, Liang Y, McCormack M, Montgomery HE, Pan H, Robbins PA, Shianna KV, Tam SC, Tsering N, Veeramah KR, Wang W, Wangdui P, Weale ME, Xu Y, Xu Z, Yang L, Zaman MJ, Zeng C, Zhang L, Zhang X, Zhaxi P, Zheng YT: Natural selection on EPAS1 (HIF2alpha) associated with low hemoglobin concentration in Tibetan highlanders. Proc Natl Acad Sci U S A 2010, 107:11459-11464.

10. Gale DP, Harten SK, Reid CDL, Tuddenham EGD, Maxwell PH: Autosomal dominant erythrocytosis and pulmonary arterial hypertension associated with an activating HIF2a mutation. Blood 2008, 112:919-921.

11. Percy MJ: Familial erythrocytosis arising from a gain-of-function mutation in the HIF2A gene of the oxygen sensing pathway. Ulster Med J 2008, 77:86-88.

12. Henderson J, Withford-Cave JM, Duffy DL, Cole SJ, Sawyer NA, Gulbin JP, Hahn A, Trent RJ, Yu B: The EPAS1 gene influences the aerobic-anaerobic contribution in elite endurance athletes. Hum Genet 2005, 118:416-423.

13. Eynon N, Hanson ED, Lucia A, Houweling PJ, Garton F, North KN, Bishop DJ: Genes for elite power and sprint performance: ACTN3 leads the way. Sports Med 2013, 43:803-817

14. Kruk J: Good scientific practice and ethical principles in scientific research and higher education. Cent Eur J Sports Sci Med 2013, 1:25-29.

15. The Ensembl Project. [www.ensembl.org]

16. Primer3web software v. 4.0.0. [http://bioinfo.ut.ee/primer3/]

17. Little J, Higgins JPT, loannidis JPA, Moher D, Gagnon F, von Elm E, Khoury MJ, Cohen B, Davey-Smith G, Grimshaw J, Scheet P, Gwinn M, Williamson RE, Zou GY, Hutchings K, Johnson CY, Tait V, Wiens M, Golding J, van Duijn C, McLaughlin J, Paterson A, Wells G, Fortier I, Freedman M, Zecevic M, King R, Infante-Rivard C, Stewart A, Birkett N: Strengthening the reporting of genetic association studies (STREGA)-an extension of the STROBE statement. Eur J Clin Invest 2009, 39:247-266.

18. Friedman JH: Multivariate adaptive regression splines. Ann Stat 1991 19:1-141.

19. Lin H-Y, Chen YA, Tsai Y-Y, Qu X, Tseng T-S, Park JY: TRM: a powerful two-stage machine learning approach for identifying SNP-SNP interactions. Ann Hum Genet 2012, 76:53-62.

20. Zabaleta J, Su LJ, Lin H-Y, Sierra RA, Hall MC, Sartor AO, Clark PE, Hu J, Ochoa AC: Cytokine genetic polymorphisms and prostate cancer aggressiveness. Carcinogenesis 2009, 30:1358-1362.

21. Zabaleta J, Lin H-Y, Sierra RA, Hall MC, Clark PE, Sartor OA, Hu J, Ochoa AC. Interactions of cytokine gene polymorphisms in prostate cancer risk. Carcinogenesis 2008, 29:573-578.

22. Nonyane BAS, Foulkes AS: Application of two machine learning algorithms to genetic association studies in the presence of covariates. BMC Genet 2008, 9:71.

23. Benjamini $Y$, Hochberg $Y$ : Controlling the false discovery rate: a practical and powerful approach to multiple testing. J R Stat Soc Ser B Methodol 1995, 57:289-300 [B]

24. Goldstein BA, Hubbard AE, Cutler A, Barcellos LF: An application of random forests to a genome-wide association dataset: methodological considerations \& new findings. BMC Genet 2010, 11:49.

25. Nicodemus KK, Malley JD: Predictor correlation impacts machine learning algorithms: implications for genomic studies. Bioinformatics 2009, 25:1884-1890

26. Johnson AD, Handsaker RE, Pulit SL, Nizzari MM, O'Donnell CJ, De Bakker PIW: SNAP: a web-based tool for identification and annotation of proxy SNPs using HapMap. Bioinforma Oxford Engl 2008, 24:2938-2939.

27. Royston P, Sauerbrei W: Using the Bootstrap to Explore Model Stability. In Multivariable Model - Build A Pragmatic Approach to Regres Anal based Fract Polynomials Model Contin Var. 1st edition. Chichester: Wiley; 2008:186-188.
28. Heid IIM, Jackson AUA, Randall J, Winkler T: Meta-analysis identifies 13 new loci associated with waist-hip ratio and reveals sexual dimorphism in the genetic basis of fat distribution. Nat Genet 2010, 42:950.

29. Posthumus M, September AV, O'Cuinneagain D, van der Merwe $W$ Schwellnus MP, Collins M: The COL5A1 gene is associated with increased risk of anterior cruciate ligament ruptures in female participants. Am J Sports Med 2009, 37:2234-2240.

30. Clarkson PM, Devaney JM, Gordish-Dressman H, Thompson PD, Hubal MJ, Urso M, Price TB, Angelopoulos TJ, Gordon PM, Moyna NM, Pescatello LS, Visich PS, Zoeller RF, Seip RL, Hoffman EP: ACTN3 genotype is associated with increases in muscle strength in response to resistance training in women. J Appl Physiol 2005, 99:154-163.

31. He Z-H, Hu Y, Li Y-C, Gong L-J, Cieszczyk P, Maciejewska-Karlowska A, Leonska-Duniec A, Muniesa CA, Marín-Peiro M, Santiago C, Garatachea N, Eynon N, Lucia A: PGC-related gene variants and elite endurance athletic status in a Chinese cohort: a functional study. Scand J Med Sci Sports in press.

32. Lucia A, Ruiz JR, Eynon N, Birk R, Bishop DJ, Gómez-Gallego F, Santiago C: The rs 12594956 polymorphism in the NRF-2 gene is associated with top-level Spanish athlete's performance status. J Sci Med Sport 2013, 16:135-139.

33. Ruiz JR, Eynon N, Meckel Y, Fiuza-Luces C, Santiago C, Gómez-Gallego F, Oliveira J, Lucia A: GNB3 C825T polymorphism and elite athletic status: a replication study with two ethnic groups. Int J Sports Med 2011, 32:151-153.

34. Eynon N, Ruiz JR, Femia P, Pushkarev VP, Cieszczyk P, Maciejewska-Karlowska A, Sawczuk M, Dyatlov DA, Lekontsev EV, Kulikov LM, Birk R, Bishop DJ, Lucia A: The ACTN3 R577X polymorphism across three groups of elite male European athletes. PLoS One 2012, 7:e43132

35. Yang N, MacArthur DG, Gulbin JP, Hahn AG, Beggs AH, Easteal S, North K: ACTN3 genotype is associated with human elite athletic performance. Am J Hum Genet 2003, 73:627-631.

36. Eynon N, Duarte JA, Oliveira J, Sagiv M, Yamin C, Meckel Y, Goldhammer E: ACTN3 R577X polymorphism and Israeli top-level athletes. Int J Sports Med 2009, 30:695-698.

37. Royston P, Sauerbrei W: Bootstrap assessment of the stability of multivariable models. Stata J 2009, 9:547-570.

38. Scortegagna M, Ding K, Oktay Y, Gaur A, Thurmond F, Yan L-J, Marck BT, Matsumoto AM, Shelton JM, Richardson JA, Bennett MJ, Garcia JA: Multiple organ pathology, metabolic abnormalities and impaired homeostasis of reactive oxygen species in Epas1-/- mice. Nat Genet 2003, 35:331-340.

39. Scortegagna M, Morris MA, Oktay Y, Bennett M, Garcia JA: The HIF family member EPAS1/HIF-2alpha is required for normal hematopoiesis in mice. Blood 2003, 102:1634-1640.

doi:10.1186/1471-2164-15-382

Cite this article as: Voisin et al.: EPAS1 gene variants are associated with sprint/power athletic performance in two cohorts of European athletes. BMC Genomics 2014 15:382.

\section{Submit your next manuscript to BioMed Central and take full advantage of:}

- Convenient online submission

- Thorough peer review

- No space constraints or color figure charges

- Immediate publication on acceptance

- Inclusion in PubMed, CAS, Scopus and Google Scholar

- Research which is freely available for redistribution 